\title{
Labs collide over rival tritium schemes
}

Pleasanton, California. A \$2-billion prototype fusion machine has been suggested by the former director of the Lawrence Livermore National Laboratory (LLNL) in California as an alternative means of producing tritium for US nuclear weapons to the planned particle accelerator.

John Nuckolls, who pioneered inertial confinement fusion research with Edward Teller, and who was director of LLNL until 1994, says that such a machine could help to "make the transition" between the planned National Ignition Facility (NIF) - which is expected to demonstrate the feasibility of inertial confinement fusion - and a commercially viable system.

The United States says it will need a supply of tritium to replenish its nuclear weapons in about ten years, and the Los Alamos National Laboratory in New Mexico is leading a \$280-million research programme to design a particle accelerator to produce it (see Nature 377, 567; 1995). All US nuclear weapons contain tritium, which decays with a half-life of eleven years.

The accelerator being designed at Los Alamos would produce $3 \mathrm{~kg}$ of tritium a year by firing a proton beam into a tungsten target to generate neutrons, which are then absorbed in helium-3 to make the tritium. It would cost around $\$ 2$ billion to build, but would consume $400 \mathrm{MW}$ of electricity enough for a city of half a million people and could therefore cost as much as $\$ 200$ million a year to operate.

The Department of Energy has already said that it will decide in 1998 whether to build the accelerator; if it goes ahead, it would be built at Savannah River, South Carolina (see Nature 376, 201; 1995).

But Nuckolls told a meeting of Fusion Power Associates, a pro-fusion lobby group, at Pleasanton, California, two weeks ago that if a START 3 treaty is agreed with Russia and the tritium requirement is delayed, an inertial confinement fusion machine could be built instead of the accelerator.

"I know it is going to be awkward explaining this to our friends at Los Alamos," Nuckolls said. "But I think we've got to look at it." Los Alamos and Livermore have been feuding about projects and prestige ever since Teller set up the California lab in 1952.

Nuckolls suggests that the prototype machine could use either a solid-state laser or a beam of heavy ions to induce fusion in tiny pellets of deuterium fuel. The pellets might be contained in a small cylinder. This converts the beams into intense X-rays, which compress the pellet to induce fusion.

He envisages a machine which would do this every two seconds, using a pulse of 5 megajoules of energy each time to induce 40 times as much energy by fusion. The laser or ion beam could be built for $\$ 1$ billion, he argues, leaving $\$ 100$ million for a factory to produce the pellets and $\$ 900$ million for the equipment to contain the fusion blasts.

Several experts at the meeting said that most of the energy is given off as heat by the neutrons as they are absorbed, and containment is therefore not regarded as a major technical challenge.

Paul Lisowski, head of the Accelerator Production of Tritium programme at Los Alamos, said of Nuckolls' proposal: "It is

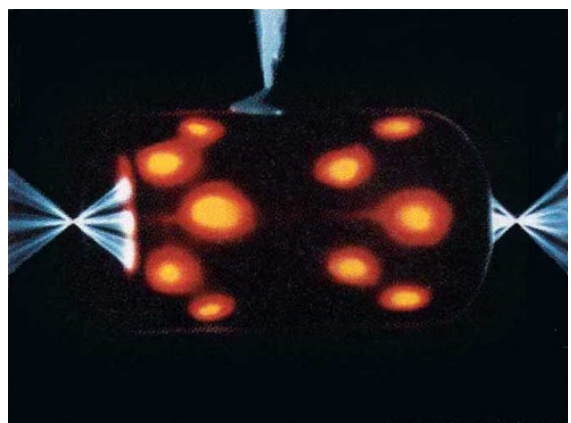

Focusing the debate: a laser target Hohlraum, which creates fusion by reflecting $X$-rays onto a one-millimetre fuel capsule.

possible that the [inertial confinement fusion] technology will be very productive in the future - but it is not the kind of proven technology which you could use to produce tritium for national security purposes."

The proposed 40-fold 'gain' for Nuckolls' machine would be four times as great as that expected from the \$1-billion National Ignition Facility (NIF), which is being designed at Lawrence Livermore. NIF is funded as part of the stockpile stewardship programme, which looks after nuclear weapons. But its main scientific task will be to establish that inertial confinement fusion can be induced under controlled circumstances.

\section{Cancer genetics group drafts guidelines}

Washington. Women found to have genetic mutations predisposing them to breast cancer should begin having annual mammograms between the ages of 25 and 35 , according to a draft report by a US National Institutes of Health (NIH) task force due to be published soon.

This and other recommendations on the management of asymptomatic carriers of cancer-predisposing mutations result from a year-long study by a task force of the NIH Cancer Genetics Studies Consortium.

Wylie Burke, an associate professor of medicine at the University of Washington in Seattle, and chair of the task force, outlined the recommendations at a meeting last month of the Advisory Council to the National Center for Human Genome Research.

The recommendations deal with surveillance and prophylactic surgery in healthy carriers of mutations predisposing to
NIF will test out pellets which are ignited directly and indirectly, inside Hohlraums, but it will operate slowly, its optical systems taking hours to cool down after each "shot".

The Clinton administration intends to announce its final decision to build NIF at Lawrence Livermore just before November's presidential election, in which Californian votes will play a critical role.

The principles of inertial confinement fusion, including the role of the Hohlraum, were classified in the United States until 1993. Declassification has given fresh impetus to the concept, at a time when the alternative fusion technology - magnetic fusion, which involves containing a ring of hot plasma gas in a ring or tokamak - is struggling to prove its feasibility as a potential energy source.

The chief advantage of inertial confinement fusion is that, on the basis of results from classified underground tests, physicists are already confident that it will work. But it is at an early stage of development at a time when no money is available to back new energy sources. Critics also predict that an energy source based on repeated, albeit very small, thermonuclear explosions, will lack public appeal.

Nuckolls says the inertial confinement fusion community has been "unified as never before" under the stockpile stewardship programme. But he predicts that stockpile stewardship will come under funding pressure, and that defence money will be unavailable for a machine after NIF, unless it has a specific defence function such as tritium production or the destruction of plutonium.

He describes his own idea as "out-of-thebox thinking", adding that "the community will have to engage in more of that" if it is to progress after NIF.

Colin Macilwain

breast, ovarian, prostate, colon and endometrial cancers. In addition to early mammography for $B R C A 1$ or $B R C A 2$ mutation carriers, for example, the draft recommends that the former should undergo pelvic examination and transvaginal ultrasound every six to 12 months, beginning between the ages of 25 and 35, to detect ovarian cancer.

Burke says the recommendations are based on "scanty" and "lacking" information in a field greatly in need of more research. For example, there have been no studies of the effectiveness of early mammography in mutation carriers. "We are doing the best we can with the information at hand," she says. "The most important issue is to think through [current] research efforts to ensure that they produce the kind of information we were looking for and couldn't find."

Meredith Wadman 